\title{
ON THE DISTRIBUTION OF FREE PATH LENGTHS FOR THE PERIODIC LORENTZ GAS III
}

\author{
EMANUELE CAGLIOTI AND FRANÇOIS GOLSE
}

\begin{abstract}
For $r \in(0,1)$, let $Z_{r}=\left\{x \in \mathbf{R}^{2} \mid \operatorname{dist}\left(x, \mathbf{Z}^{2}\right)>r / 2\right\}$ and $\tau_{r}(x, v)=\inf \left\{t>0 \mid x+t v \in \partial Z_{r}\right\}$. Let $\Phi_{r}(t)$ be the probability that $\tau_{r}(x, v) \geq t$ for $x$ and $v$ uniformly distributed in $Z_{r}$ and $\mathbf{S}^{1}$ respectively. We prove in this paper that

$$
\begin{aligned}
& \limsup _{\epsilon \rightarrow 0^{+}} \frac{1}{|\ln \epsilon|} \int_{\epsilon}^{1 / 4} \Phi_{r}\left(\frac{t}{r}\right) \frac{d r}{r}=\frac{2}{\pi^{2} t}+O\left(\frac{1}{t^{2}}\right) \\
& \liminf _{\epsilon \rightarrow 0^{+}} \frac{1}{|\ln \epsilon|} \int_{\epsilon}^{1 / 4} \Phi_{r}\left(\frac{t}{r}\right) \frac{d r}{r}=\frac{2}{\pi^{2} t}+O\left(\frac{1}{t^{2}}\right)
\end{aligned}
$$

as $t \rightarrow+\infty$. This result improves upon the bounds on $\Phi_{r}$ in Bourgain-Golse-Wennberg [Commun. Math. Phys. 190 (1998), 491-508]. We also discuss the applications of this result in the context of kinetic theory.
\end{abstract}

\section{Statement of the Problem and main Results}

1.1. The periodic Lorentz gas. Let $r \in\left(0, \frac{1}{2}\right)$ and define

$$
Z_{r}=\left\{x \in \mathbf{R}^{2} \mid \operatorname{dist}\left(x, \mathbf{Z}^{2}\right)>r / 2\right\} .
$$

Consider a point particle moving at speed 1 inside $Z_{r}$ and being specularly reflected each time it meets the boundary of $Z_{r}$. Such a dynamical system is referred to as "a periodic, two-dimensional Lorentz gas". (Indeed, Lorentz used the methods of kinetic theory to describe the motion of electrons in a metal as that of a collisionless gas of point particles bouncing on the crystalline structure of atoms in the metal [13]).

The "free path length" (or "(forward) exit time") starting from $x \in$ $Z_{r}$ in the direction $v \in \mathbf{S}^{1 "}$ is defined as

$$
\tau_{r}(x, v)=\inf \left\{t>0 \mid x+t v \in \partial Z_{r}\right\}, \quad(x, v) \in Z_{r} \times \mathbf{S}^{1} .
$$

For each $v=\left(v_{1}, v_{2}\right) \in \mathbf{S}^{1}$ such that $v_{1} v_{2} \neq 0$ and the ratio $v_{1} / v_{2} \in$ $\mathbf{R} \backslash \mathbf{Q}$, one has $\tau_{r}(x, v)<+\infty$, since any orbit of a linear flow with irrational slope on the 2-torus is dense (see for instance in [1], - section 51, corollary 1, p. 287 - for this well-known fact).

Set $Y_{r}=Z_{r} / \mathbf{Z}^{2}$; since $\tau_{r}(x, v)=\tau_{r}(x+k, v)$ for each $(x, v) \in Z_{r} \times \mathbf{S}^{1}$ and $k \in \mathbf{Z}^{2}$, the function $\tau_{r}$ can be seen as defined on $Y_{r} \times \mathbf{S}^{1}$. 

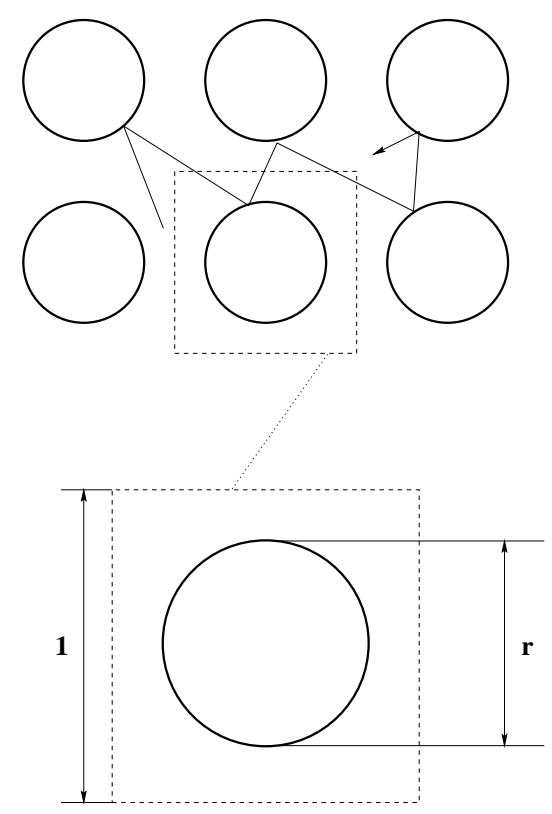

Figure 1. The Lorentz gas: $Z_{r}$ and the punctured torus $Y_{r}$

\subsection{Invariant measure for the Lorentz gas. Let}

$$
V_{r}=d x d v-\operatorname{meas}\left(Y_{r} \times \mathbf{S}^{1}\right) \text { and } \mu_{r}=\frac{1}{V_{r}} d x d v .
$$

Thus $\mu_{r}$ is a Borelian probability measure on $Y_{r} \times \mathbf{S}^{1}$.

On the other hand, the evolution of the Lorentz gas is governed by the broken Hamiltonian flow

$$
\begin{array}{ll}
(\dot{x}(t), \dot{v}(t))=(v(t), 0), & \text { whenever } x(t) \in Y_{r}, \\
v\left(t^{+}\right)=v\left(t^{-}\right)-2 v\left(t^{-}\right) \cdot n_{x(t)} n_{x(t)}, & \text { whenever } x(t) \in \partial Y_{r},
\end{array}
$$

where $n_{x}$ denotes the inward unit normal to $\partial Y_{r}$ at point $x$. As can be easily checked, the measure $\mu_{r}$ is invariant under the flow (1.3).

Let $m \in L^{\infty}\left(\mathbf{S}^{1}\right)$ such that

$$
m \geq 0 \text { and } \iint_{Y_{r} \times \mathbf{S}^{1}} m(\theta) d \mu_{r}(x, \theta)=1 .
$$

The object of interest in the present paper is the distribution of $\tau_{r}$ under $m d \mu_{r}$, ie. the function $\Phi_{r}^{m}: \mathbf{R}_{+} \rightarrow[0,1]$ defined by

$$
\Phi_{r}^{m}(t)=m d \mu_{r}-\operatorname{Prob}\left(\left\{(x, v) \in Y_{r} \times \mathbf{S}^{1} \mid \tau_{r}(x, v)>t\right\}\right) .
$$

More precisely, we are interested in the asymptotic behavior of $\Phi_{r}^{m}(t)$ as $r \rightarrow 0^{+}$and for large values of $t$ - large compared to $1 / r$. 
As explained in 7] pp. 221-222, knowing the distribution of the free path lengths in the small $r$ limit has several important implications. For instance, it leads to the correct asymptotic model for the Boltzmann-Grad limit of the periodic Lorentz gas - which is not governed by the linear Boltzmann equation (equation (10) of [13]): see below. Another application bears on the asymptotic behavior of the Kolmogorov-Sinai entropy — or equivalently, of the Lyapunov exponent — of the periodic Lorentz gas in the small $r$ limit. We refer to [9] for a survey of the most recent results and open questions on this subject, and to [8] which addresses one of these open problems by methods similar to those developed here.

1.3. Main result. The reference [6 established for each $m \in L^{\infty}\left(\mathbf{S}^{1}\right)$ as in (1.4) the existence of two positive constants $C_{m}$ and $C_{m}^{\prime}$ such that, for each $r \in\left(0, \frac{1}{2}\right)$ and each $t>1 / r$

$$
\frac{C_{m}}{r t} \leq \Phi_{r}^{m}(t) \leq \frac{C_{m}^{\prime}}{r t}
$$

(see Theorems B and C in [6]). In (1.6), the upper bound was proved by an argument based on Fourier series, while the lower bound was obtained by a geometric construction exhausting all possible channels, ie. infinitely long open strips included in $Z_{r}$. The validity of the lower bound in (1.6) was extended to arbitrary space dimensions in [12].

Numerical simulations in [12] suggest the following questions: for each $m \in L^{\infty}\left(\mathbf{S}^{1}\right)$ as in (1.4) does one have, for each $t$ large enough (say, for each $t>2$ )

$$
\Phi_{r}^{m}\left(\frac{t}{r}\right) \rightarrow \Lambda^{m}(t) \text { as } \epsilon \rightarrow 0 ?
$$

and, if so, does one have, for some constant $C>0$

$$
\Lambda^{m}(t) \sim \frac{C}{t} \text { as } t \rightarrow+\infty ?
$$

We have not been able to fully answer (1.7), but, were (1.7) true, our main result in this paper (Theorem 1.1 below) would answer (1.8) by giving an explicit value for $C$. It confirms the numerical results obtained in [12] (see figures 4 and 5 there). Throughout the paper, the notation for the convergence in the sense of Cesaro is as follows:

$$
C-\limsup _{\epsilon \rightarrow 0^{+}} f(\epsilon)=l \text { means that } \limsup _{\epsilon \rightarrow 0^{+}} \frac{1}{|\ln \epsilon|} \int_{\epsilon}^{\epsilon^{*}} f(r) \frac{d r}{r}=l
$$

for some $\epsilon^{*}>0$. (A similar notation is used for the liminf and the lim in the sense of Cesaro). 
Theorem 1.1. Let $m \in L^{\infty}\left(\mathbf{S}^{1}\right)$ satisfying $\left.\sqrt{1.4}\right)$. Let $t^{*}>\sqrt{2}$ and let

$$
\Lambda_{+}^{m}\left(t^{*}\right)=C-\limsup _{r \rightarrow 0^{+}} \Phi_{r}^{m}\left(\frac{t^{*}}{r}\right), \text { and } \Lambda_{-}^{m}\left(t^{*}\right)=C \liminf _{r \rightarrow 0^{+}} \Phi_{r}^{m}\left(\frac{t^{*}}{r}\right) \text {. }
$$

Then

$$
\Lambda_{+}^{m}\left(t^{*}\right) \sim \frac{2}{\pi^{2} t^{*}}, \text { and } \Lambda_{-}^{m}\left(t^{*}\right) \sim \frac{2}{\pi^{2} t^{*}} \text { as } t^{*} \rightarrow+\infty .
$$

More precisely,

$$
\left|\Lambda_{ \pm}^{m}\left(t^{*}\right)-\frac{2}{\pi^{2} t^{*}}\right| \leq \frac{8\|m\|_{L^{\infty}}}{t^{*}-3} .
$$

A serious shortcoming of the result above is the need for averaging in $r$ before letting $r \rightarrow 0^{+}$. It seems however that it cannot be avoided, at least by using the techniques of the present paper.

This leads to a natural question, that of the choice of the measure $\frac{d r}{r}$ to define the Cesaro mean in Theorem 1.1. The reasons for this choice are made clear by following the proof, but we take this opportunity of giving an idea of this proof by providing some qualitative argument in favor of this choice.

The proof of Theorem 1.1 is based on comparing the size $r$ of the obstacle with the sequence of errors $d_{n}$ in the approximation by continued fractions of $v_{2} / v_{1}$, ie. of the slope of the linear flow (say, in the case where $0<v_{2}<v_{1}$ ). It is natural in this context to renormalize the problem by applying to $\alpha=v_{2} / v_{1}$ the Gauss map $T: x \mapsto \frac{1}{x}-\left[\frac{1}{x}\right]-$ we refer to the appendix for more details on these notions. Lemma 7.1 below and especially the formula $d_{n}(\alpha)=\alpha d_{n-1}(T(\alpha))$ show that the exit time problem with slope $\alpha$ and obstacle of size $r$ is mapped to the analogous problem with slope $T \alpha$ and obstacle of size $\alpha r$. Hence it is natural to define the Cesaro average in Theorem 1.1 with the measure $\frac{d r}{r}$ which is the scale invariant (Haar) measure of the multiplicative group $\mathbf{R}_{+}^{*}$.

Remark 1.1. In fact, one can prove that $\Lambda_{+}^{m}\left(t^{*}\right)=\Lambda_{-}^{m}\left(t^{*}\right)$ for each $t^{*}$ large enough (say, for $t^{*}>10$ ). (In other words $C-\lim _{r \rightarrow 0^{+}} \Phi_{r}^{m}\left(\frac{t^{*}}{r}\right)$ exists). However, this result requires some significant improvements of the method used in the present paper; they will be described in [8].

\section{A partition of $\mathbf{T}^{2}$}

In 1989, R. Thom posed the following problem:

- What is the longest orbit of a linear flow with irrational slope on a flat torus with a disk removed? 


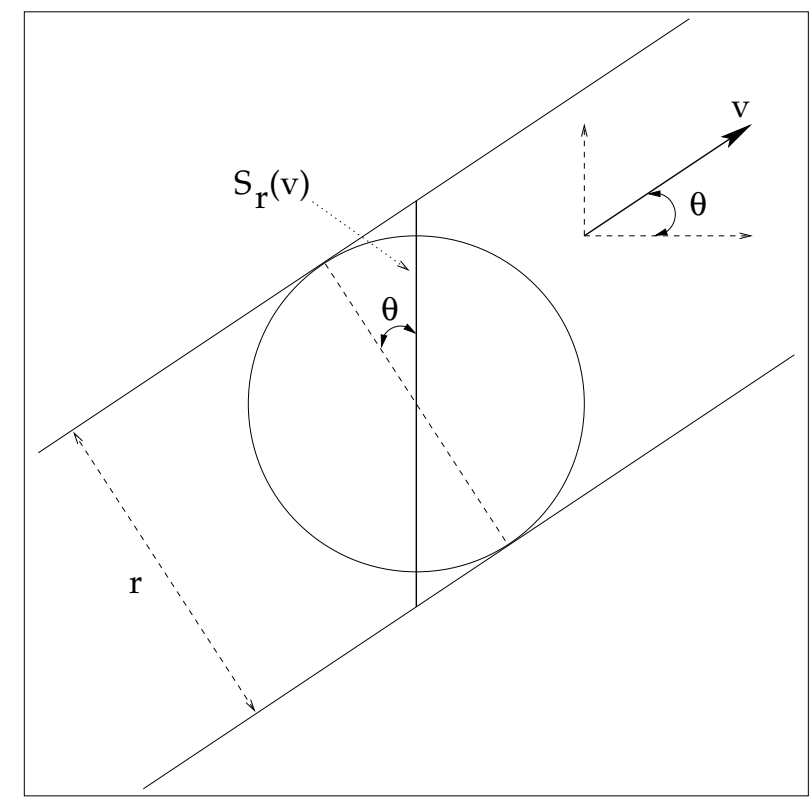

Figure 2. The punctured torus $Y_{r}$ and the slit $S_{r}(v)$

The answer to this question was found by Blank and Krikorian in 2] and is summarized as follows. Without loss of generality, assume that the linear flow is $x \mapsto x+t v$ where $v=(\cos \theta, \sin \theta)$ with $\theta \in\left(0, \frac{\pi}{4}\right)$. The removed disk of diameter $r$ is replaced by a vertical slit $S_{r}(v)$ of length $r / \cos \theta$ with the same center (see figure 2).

Proposition 2.1 (Blank-Krikorian 2] p. 722). Let $r \in\left(0, \frac{1}{2}\right), \theta \in$ $\left(0, \frac{\pi}{4}\right)$ and $v=(\cos \theta, \sin \theta)$. Assume that $\tan \theta$ is irrational. There exist three positive numbers $l_{A}(r, v), l_{B}(r, v)$ and $l_{C}(r, v)$ satisfying

$$
l_{A}(r, v)<l_{B}(r, v) \quad \text { and } \quad l_{C}(r, v)=l_{A}(r, v)+l_{B}(r, v)
$$

and such that, for any orbit $\gamma$ of the linear flow $x \mapsto x+t v$ in $\mathbf{T}^{2} \backslash S_{r}(v)$, length $(\gamma)$ takes one of the three values $l_{A}(r, v), l_{B}(r, v)$ or $l_{C}(r, v)$.

Conversely, given any $l \in\left\{l_{A}(r, v), l_{B}(r, v), l_{C}(r, v)\right\}$, there exists an orbit $\gamma$ of the flow $x \mapsto x+t v$ of length $l$.

Let $v$ be fixed; orbits of length $l_{A}(r, v)$ (resp. of length $l_{B}(r, v)$, $\left.l_{C}(r, v)\right)$ are referred to as orbits of type $A$ (resp. of type $\left.B, C\right)^{1}$.

\footnotetext{
${ }^{1}$ On p. 722 of [2], the sentence "If the moving slit first meets the fixed slit at the bottom, the $A$ and $B$-orbits are reversed" might be the source of a slight ambiguity in the definition of the partition above. In the present paper, the orbits of type $A$ are the shortest, consistently with the table on p. 726 of [2]. Hence the roles of orbits of type $A$ and $B$ cannot be reversed in the present discussion.
} 


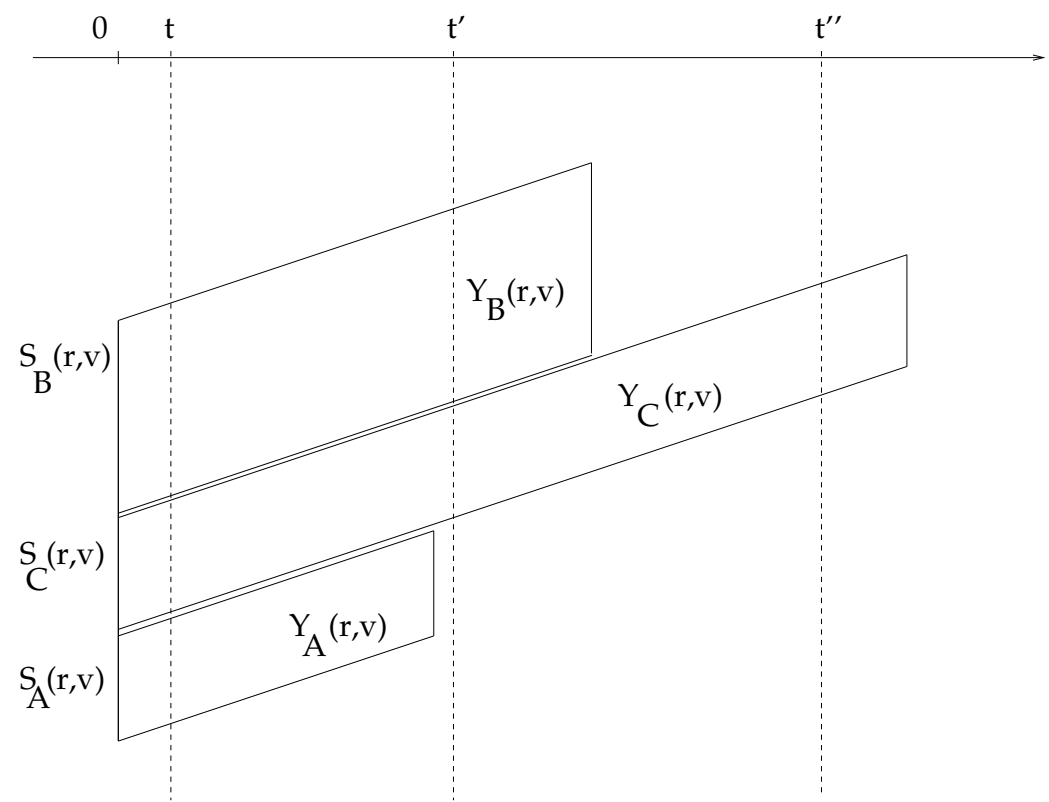

Figure 3. The partition $\left(Y_{A}(r, v), Y_{B}(r, v), Y_{C}(r, v)\right)$ of $\mathbf{T}^{2} \backslash S_{r}(v)$

Proposition 2.1 defines a partition $\left(Y_{A}(r, v), Y_{B}(r, v), Y_{C}(r, v)\right)$ of $\mathbf{T}^{2} \backslash$ $S_{r}(v)$, where

$$
Y_{A}(r, v)=\left\{x \in Y_{r} \backslash S_{r}(v) \mid x \text { belongs to an orbit of type } A\right\}
$$

and $Y_{B}(r, v)$ and $Y_{C}(r, v)$ are similarly defined. Define further

$$
S_{A}(r, v)=\left\{y \in S_{r}(v) \mid \text { the } v \text {-orbit starting from } y \text { is of type A }\right\}
$$

with analogous definitions for $S_{B}(r, v)$ and $S_{C}(r, v)$. Clearly $Y_{A}(r, v)$ (resp. $\left.Y_{B}(r, v), Y_{C}(r, v)\right)$ is metrically equivalent to a strip (parallellogram) of length $l_{A}(r, v)$ and width $\left|S_{A}(r, v)\right|$ (resp. of length $l_{B}(r, v)$, $l_{C}(r, v)$ and width $\left.\left|S_{B}(r, v)\right|,\left|S_{B}(r, v)\right|\right)$ : see figure 3 below.

Define now $\lambda_{r}$ to be the exit time in the torus with the slit (instead of the disk) removed, ie.

$$
\lambda_{r}(z, v)=\inf \left\{t>0 \mid z+t v \in S_{r}(v)\right\} \text { for each } z \in \mathbf{T}^{2} \backslash S_{r}(v) .
$$

For $v=(\cos \theta, \sin \theta)$ with $\theta \in\left(0, \frac{\pi}{4}\right)$ as above, define

$$
\psi_{r}(t, v)=d x-\operatorname{Prob}\left(\left\{z \in \mathbf{T}^{2} \backslash S_{r}(v) \mid \lambda_{r}(z, v) \geq t\right\}\right) .
$$

With the partition of $\mathbf{T}^{2} \backslash S_{r}(v)$ in $\left(Y_{A}(r, v), Y_{B}(r, v), Y_{C}(r, v)\right)$, which is metrically equivalent to the disjoint union of three strips as represented on figure 3 , computing $\psi_{r}(t, v)$ in terms of the quantities $\left|S_{A}(r, v)\right|$, 


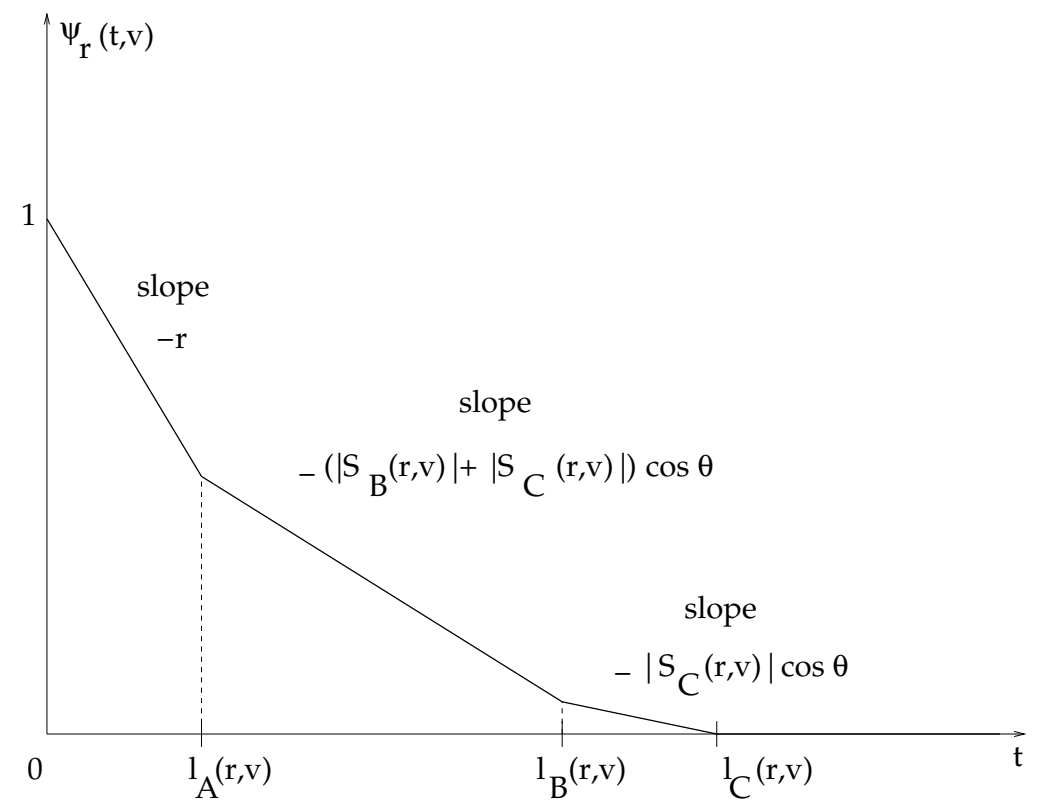

Figure 4. Graph of $t \mapsto \psi_{r}(t, v)$

$l_{A}(r, v),\left|S_{B}(r, v)\right|, l_{B}(r, v),\left|S_{C}(r, v)\right|$ and $l_{C}(r, v)$ becomes an easy task. One finds that

- if $0 \leq t \leq l_{A}(r, v)$, then

$$
\psi_{r}(t, v)=1-t r
$$

- if $l_{A}(r, v) \leq t^{\prime} \leq l_{B}(r, v)$, then

$$
\begin{aligned}
\psi_{r}\left(t^{\prime}, v\right)=1 & -l_{A}(r, v) r \\
& -\left(t^{\prime}-l_{A}(r, v)\right)\left(\left|S_{B}(r, v)\right|+\left|S_{C}(r, v)\right|\right) \cos \theta ;
\end{aligned}
$$

- if $l_{B}(r, v) \leq t^{\prime \prime} \leq l_{C}(r, v)$, then

$$
\psi_{r}\left(t^{\prime \prime}, v\right)=\left(l_{C}(r, v)-t^{\prime \prime}\right)\left|S_{C}(r, v)\right| \cos \theta ;
$$

- if $t \geq l_{C}(r, v)$, then

$$
\psi_{r}(t, v)=0 .
$$

The graph of $t \mapsto \psi_{r}(t, v)$ is presented on figure 4 below.

So far, the distribution $\psi_{r}(t, v)$ has been computed in terms of the quantities $\left|S_{A}(r, v)\right|, l_{A}(r, v),\left|S_{B}(r, v)\right|, l_{B}(r, v),\left|S_{C}(r, v)\right|$ and $l_{C}(r, v)$ that characterize the partition $\left(Y_{A}(r, v), Y_{B}(r, v), Y_{C}(r, v)\right)$ of $\mathbf{T}^{2} \backslash S_{r}(v)$. These quantities can be expressed (see [2]) in terms of the continued fraction expansion of $\tan \theta$, in the following manner.

In the following discussion, we freely use the notations recalled in the Appendix below. Let $\alpha=\tan \theta \in(0,1)$; the $\theta$ 's for which $\alpha \in \mathbf{Q}$ 
form a set of measure 0 and are discarded in the argument below. With the sequence of errors $d_{n}$ in the continued fraction expansion of $\alpha$, we consider the following partition of the interval $(0,1)$ :

$$
(0,1)=\bigcup_{n \geq 1} I_{n}, \quad \text { with } I_{n}=\left[d_{n}, d_{n-1}\right) .
$$

Each interval $I_{n}$ is further partitioned into

$$
I_{n}=\bigcup_{1 \leq k \leq a_{n}} I_{n, k}, \quad \text { with } I_{n, k}=\left[\sup \left(d_{n}, d_{n-1}-k d_{n}\right), d_{n-1}-(k-1) d_{n}\right),
$$

so that eventually we arrive at the following nested partition of $(0,1)$ :

$$
(0,1)=\bigcup_{n \geq 1} \bigcup_{1 \leq k \leq a_{n}} I_{n, k} .
$$

Proposition 2.2 (Blank-Krikorian [2] p. 726). Let $\theta \in\left(0, \frac{\pi}{4}\right)$ and $r \in\left(0, \frac{1}{2}\right)$. Let $v=(\cos \theta, \sin \theta)$ and set $\alpha=\tan \theta$ (it is assumed that $\alpha \notin \mathbf{Q})$. For $R=\frac{r}{\cos \theta}$, the integers $n \geq 1$ and $k$ such that $1 \leq k \leq a_{n}$ ( $a_{n}$ being the $n$-th term in the continued fraction expansion of $\alpha$ ) are defined by the fact that $R \in I_{n, k}$ (since the intervals $I_{n, k}$ form a partition of $(0,1)$ : see (2.9)). Then

- $l_{A}(r, v)=q_{n}$ and $\left|S_{A}(r, v)\right|=R-d_{n}$,

- $l_{B}(r, v)=q_{n-1}+k q_{n}$ and $\left|S_{B}(r, v)\right|=R-\left(d_{n-1}-k d_{n}\right)$,

- $l_{C}(r, v)=q_{n-1}+(k+1) q_{n}$ and $\left|S_{C}(r, v)\right|=d_{n-1}-(k-1) d_{n}-R$.

Using these values, we arrive at the following expression for $\psi_{r}(t, v)$, whenever $R=\frac{r}{\cos \theta} \in I_{n, k}$ (see figure 5 below):

- if $0 \leq t \cos \theta \leq q_{n}$, then

$$
\psi_{r}(t, v)=1-t r
$$

- if $q_{n} \leq t \cos \theta \leq q_{n-1}+k q_{n}$, then

$$
\psi_{r}(t, v)=1-R q_{n}-d_{n}\left(t \cos \theta-q_{n}\right),
$$

- if $q_{n-1}+k q_{n} \leq t \cos \theta \leq q_{n-1}+(k+1) q_{n}$, then

$$
\begin{aligned}
\psi_{r}(t, v)=1 & -R q_{n}-d_{n}\left[q_{n-1}+(k-1) q_{n}\right] \\
& -\left[R-\left(d_{n-1}-(k-1) d_{n}\right)\right]\left(t \cos \theta-q_{n-1}-k q_{n}\right),
\end{aligned}
$$

- if $t \cos \theta \geq q_{n-1}+(k+1) q_{n}$, then

$$
\psi_{r}(t, v)=0 \text {. }
$$

Finally, the discussion above leads to the statistics of the exit time $\lambda_{r}(z, v)$ defined in (2.1) corresponding to the torus $\mathbf{T}^{2}$ with the slit $S_{r}(v)$ removed. In the case of the torus with the disk removed, the 


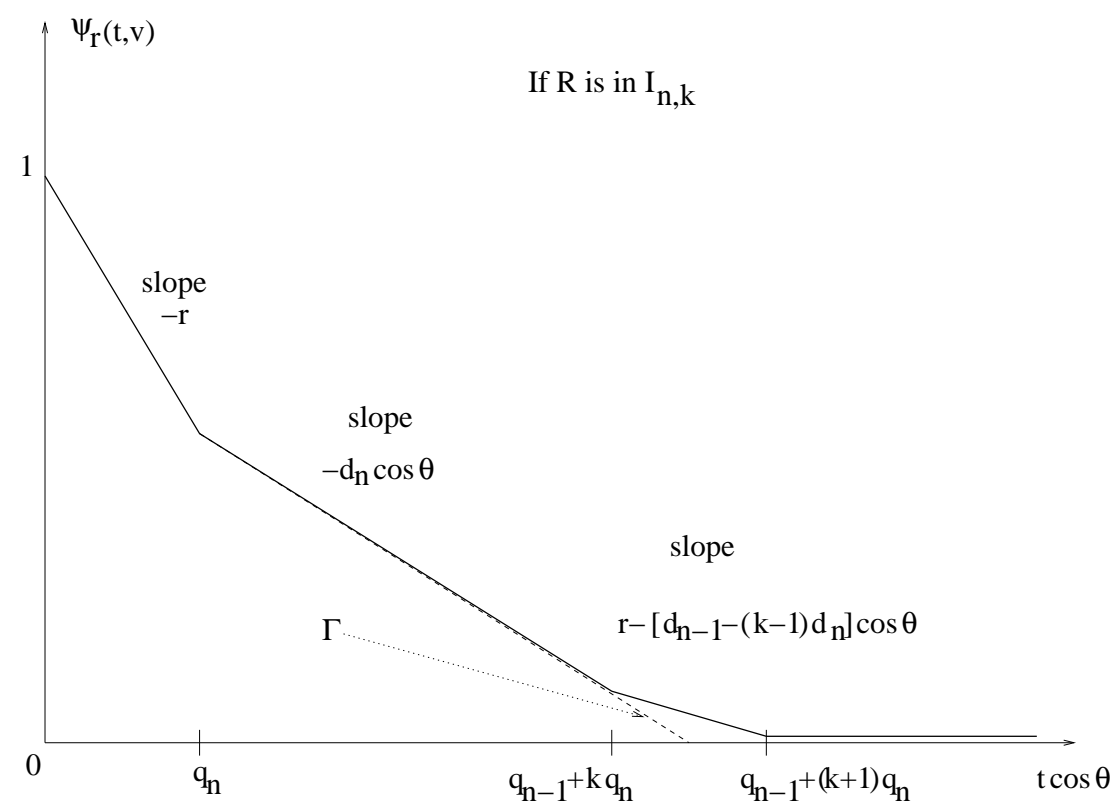

Figure 5. Graph of $t \mapsto \psi_{r}(t, v)$ for $R \in I_{n, k}$

corresponding exit time $\tau_{r}(x, v)$ defined in (1.2) is related to $\lambda_{r}(z, v)$ by the obvious inequalities

$$
\lambda_{r}(x, v)-\frac{r}{2} \leq \tau_{r}(x, v) \leq \lambda_{r}(x, v)+\frac{r}{2} \text { for each } x \in Y_{r} \backslash S_{r}(v) .
$$

Define

$$
\phi_{r}(t, v)=d x-\operatorname{Prob}\left(\left\{x \in Y_{r} \backslash S_{r}(v) \mid \tau_{r}(x, v) \geq t\right\}\right) ;
$$

because of (2.14), one has

$$
\psi_{r}\left(t+\frac{r}{2}, v\right) \leq \phi_{r}(t, v) \leq \psi_{r}\left(t-\frac{r}{2}, v\right), \quad t \geq \frac{r}{2} .
$$

The remaining part of the paper uses the evaluation of $\phi_{r}$ based on this inequality together with the formulas (2.10), (2.11), (2.12) and (2.13) for $\psi_{r}$.

\section{AN ERGODIC THEOREM}

Given $\alpha \in(0,1)$ and $\epsilon \in(0,1)$, we define

$$
N(\alpha, \epsilon)=\inf \left\{n \in \mathbf{N} \mid d_{n+1}(\alpha)<\epsilon\right\} .
$$

In terms of the partition (2.7) of $(0,1), N(\alpha, \epsilon)$ can be equivalently defined by the condition

$$
\epsilon \in I_{N(\alpha, \epsilon)+1} .
$$

We start by recalling the following more or less classical lemma. 
Lemma 3.1. For ae. $\alpha \in(0,1)$, one has

$$
N(\alpha, \epsilon) \sim-\frac{12 \ln 2}{\pi^{2}} \ln \epsilon \quad \text { as } \epsilon \rightarrow 0^{+} .
$$

Proof. The definition of $N(\alpha, \epsilon)$ and the third formula in Lemma 7.1 imply that

$$
\sum_{j=0}^{N(\alpha, \epsilon)-1}-\ln T^{j} \alpha \leq-\ln \epsilon<\sum_{j=0}^{N(\alpha, \epsilon)}-\ln T^{j} \alpha,
$$

where $T$ is the Gauss map (17.1). First we prove that $N(\alpha, \epsilon) \rightarrow+\infty$ as $\epsilon \rightarrow 0^{+}$ae. in $\alpha \in(0,1)$. Indeed, let $C>0$ and let

$$
E_{C}=\left\{\alpha \in(0,1) \mid N(\alpha, \epsilon) \leq C, \text { for all } \epsilon \in\left(0, \frac{1}{2}\right)\right\} .
$$

For all $\alpha \in E_{C}$ and all $\epsilon \in\left(0, \frac{1}{2}\right)$

$$
-\ln \epsilon \leq \sum_{j=0}^{C}-\ln T^{j} \alpha
$$

therefore, for all $\epsilon \in\left(0, \frac{1}{2}\right)$,

$$
-\ln \epsilon \cdot d g-\operatorname{meas}\left(E_{C}\right) \leq(C+1) \int_{0}^{1}(-\ln \alpha) d g(\alpha)<+\infty,
$$

since the measure $d g$ in (7.2) is invariant under $T$. This implies that

$$
\text { for each } C>0, d g-\text { meas }\left(E_{C}\right)=0 \text {. }
$$

Because $N(\alpha, \epsilon)$ is a nonincreasing function of $\epsilon$,

$$
\text { for each } \alpha \in\left(\bigcup_{m \geq 1} E_{m}\right)^{c}, N(\alpha, \epsilon) \rightarrow+\infty
$$

as $\epsilon \rightarrow 0^{+}$and

$$
d g-\operatorname{meas}\left(\bigcup_{m \geq 1} E_{m}\right)=0 .
$$

Secondly, by Birkhoff's ergodic theorem, there exists a $d g$-negligeable set $E^{\prime}$ such that

$$
\text { for each } \alpha \in\left(E^{\prime}\right)^{c}, \quad \frac{1}{N} \sum_{0}^{N-1}-\ln T^{j} \alpha \rightarrow \int_{0}^{1}(-\ln \alpha) d g(\alpha)
$$

as $N \rightarrow+\infty$, since the Gauss transformation $T$ of $(0,1)$ is ergodic with respect to the invariant measure $d g(\alpha)$ - see the Appendix below. 
Hence

$$
\begin{aligned}
& \text { for each } \alpha \in\left(E^{\prime} \cup \bigcup_{m \geq 1} E_{m}\right)^{c}, \\
& \frac{1}{N(\alpha, \epsilon)} \sum_{0}^{N(\alpha, \epsilon)-1}-\ln T^{j} \alpha \rightarrow \int_{0}^{1}(-\ln \alpha) d g(\alpha) .
\end{aligned}
$$

By (3.3), (3.5) and (3.6), one finally obtains that, as $\epsilon \rightarrow 0^{+}$,

for each $\alpha \in\left(E^{\prime} \cup \bigcup_{m \geq 1} E_{m}\right)^{c}, \quad \frac{-\ln \epsilon}{N(\alpha, \epsilon)} \rightarrow \int_{0}^{1}(-\ln \alpha) d g(\alpha)=\frac{\zeta(2)}{2 \ln 2}$

(replacing the $\ln$ under the integral sign by its Taylor series at $\alpha=$ $1)$.

The main result of this section is the following application of the Birkhoff ergodic theorem. We shall need the notations below:

$$
\begin{aligned}
& \Delta_{0}(\alpha, x)=-x-\ln d_{N\left(\alpha, e^{-x}\right)+1}(\alpha), \\
& \Delta_{1}(\alpha, x)=-x-\ln d_{N\left(\alpha, e^{-x}\right)}(\alpha) .
\end{aligned}
$$

Proposition 3.1. Let $f$ be a bounded nonnegative measurable function on $\mathbf{R}^{2}$. For each $x^{*} \in \mathbf{R}$ and ae. in $\alpha \in(0,1)$, one has

$$
\frac{1}{|\ln \epsilon|} \int_{x^{*}}^{|\ln \epsilon|} f\left(\Delta_{0}(\alpha, x), \Delta_{1}(\alpha, x)\right) d x \rightarrow \frac{12}{\pi^{2}} \int_{0}^{1} \frac{F(\theta) d \theta}{1+\theta}
$$

as $\epsilon \rightarrow 0$, where

$$
F(\theta)=\int_{0}^{|\ln (\theta)|} f(|\ln (\theta)|-y,-y) d y .
$$

Proof. First we decompose the integral

$$
\begin{aligned}
\int_{x^{*}}^{|\ln \epsilon|} f\left(\Delta_{0}(\alpha, x),\right. & \left.\Delta_{1}(\alpha, x)\right) d x \\
& =\int_{x^{*}}^{0} f\left(\Delta_{0}(\alpha, x), \Delta_{1}(\alpha, x)\right) d x \\
& +\int_{\left|\ln d_{N(\alpha, \epsilon)}(\alpha)\right|}^{|\ln \epsilon|} f\left(\Delta_{0}(\alpha, x), \Delta_{1}(\alpha, x)\right) d x \\
& +\sum_{l=0}^{N(\alpha, \epsilon)-1} \int_{\left|\ln d_{l}(\alpha)\right|}^{\left|\ln d_{l+1}(\alpha)\right|} f\left(\Delta_{0}(\alpha, x), \Delta_{1}(\alpha, x)\right) d x
\end{aligned}
$$


Now observe that, whenever $x$ belongs to the domain of integration of

$$
\int_{\left|\ln d_{l}(\alpha)\right|}^{\left|\ln d_{l+1}(\alpha)\right|} f\left(\Delta_{0}(\alpha, x), \Delta_{1}(\alpha, x)\right) d x,
$$

in other words, whenever

$$
\left|\ln d_{l}(\alpha)\right|<x \leq\left|\ln d_{l+1}(\alpha)\right|, \quad \text { then } N\left(\alpha, e^{-x}\right)=l .
$$

This implies that

$$
\begin{aligned}
\int_{\left|\ln d_{l}(\alpha)\right|}^{\left|\ln d_{l+1}(\alpha)\right|} & f\left(\Delta_{0}(\alpha, x), \Delta_{1}(\alpha, x)\right) d x \\
& =\int_{-\ln d_{l}(\alpha)}^{-\ln d_{l+1}(\alpha)} f\left(-x-\ln d_{l+1}(\alpha),-x-\ln d_{l}(\alpha)\right) d x \\
& =\int_{0}^{\ln \left(d_{l}(\alpha) / d_{l+1}(\alpha)\right)} f\left(\ln \left(d_{l}(\alpha) / d_{l+1}(\alpha)\right)-y,-y\right) d y \\
& =F\left(T^{l} \theta\right) .
\end{aligned}
$$

Likewise, since $f$ is nonnegative

$$
\begin{aligned}
0 \leq \int_{\left|\ln d_{N(\alpha, \epsilon)}(\alpha)\right|}^{|\ln \epsilon|} f\left(\Delta_{0}(\alpha, x),\right. & \left.\Delta_{1}(\alpha, x)\right) d x \\
& \leq \int_{\left|\ln d_{N(\alpha, \epsilon)}(\alpha)\right|}^{\left|\ln d_{N(\alpha, \epsilon)+1}(\alpha)\right|} f\left(\Delta_{0}(\alpha, x), \Delta_{1}(\alpha, x)\right) d x \\
& \leq F\left(T^{N(\alpha, \epsilon)} \alpha\right) .
\end{aligned}
$$

Using (3.8) and (3.9) leads to

$$
\begin{aligned}
\frac{1}{|\ln \epsilon|} \int_{x^{*}}^{|\ln \epsilon|} f\left(\Delta_{0}(\alpha, x),\right. & \left.\Delta_{1}(\alpha, x)\right) d x \\
& =\frac{N(\alpha, \epsilon)}{|\ln \epsilon|}\left(\frac{1}{N(\alpha, \epsilon)} \sum_{l=0}^{N(\alpha, \epsilon)} F\left(T^{l} \theta\right)\right) \\
& =\frac{1}{|\ln \epsilon|} \int_{x^{*}}^{0} f\left(\Delta_{0}(\alpha, x), \Delta_{1}(\alpha, x)\right) d x \\
& +\frac{1}{|\ln \epsilon|} \int_{\left|\ln d_{N(\alpha, \epsilon)}(\alpha)\right|}^{|\ln \epsilon|} f\left(\Delta_{0}(\alpha, x), \Delta_{1}(\alpha, x)\right) d x .
\end{aligned}
$$

Observe first that, by its definition

$$
0 \leq F(\theta) \leq\|f\|_{L^{\infty}}|\ln \theta| \text { which implies that } F \in L^{1}((0,1), d g) .
$$


Birkhoff's ergodic theorem applied to the Gauss transformation $T$ together with Lemma 3.1 shows that

$$
\frac{1}{N(\alpha, \epsilon)} \sum_{l=0}^{N(\alpha, \epsilon)} F\left(T^{l} \theta\right) \rightarrow \frac{1}{\ln 2} \int_{0}^{1} F(\theta) d g(\theta)
$$

for ae. $\alpha \in(0,1)$ as $\epsilon \rightarrow 0$, so that the first term in the right hand side of (3.11) converges to

$$
\frac{12}{\pi^{2}} \int_{0}^{1} F(\theta) d g(\theta)
$$

as $\epsilon \rightarrow 0^{+}$ae. in $\alpha$. The second term in the right hand side of (3.11) obviously vanishes ae. in $\alpha \in(0,1)$ as $\epsilon \rightarrow 0$. As for the third term, because of (3.10), one has

$$
\begin{aligned}
0 \leq \frac{1}{|\ln \epsilon|} \int_{\left|\ln d_{N(\alpha, \epsilon)}(\alpha)\right|}^{|\ln \epsilon|} f\left(\Delta_{0}(\alpha, x),\right. & \left.\Delta_{1}(\alpha, x)\right) d x \\
& \leq \frac{N(\alpha, \epsilon)}{|\ln \epsilon|} \frac{1}{N(\alpha, \epsilon)} F\left(T^{N(\alpha, \epsilon)} \alpha\right)
\end{aligned}
$$

and the right hand side of the above inequality converges to 0 ae. in $\alpha \in(0,1)$ as $\epsilon \rightarrow 0^{+}$by Birkoff's ergodic theorem and Lemma 3.1 .

\section{Proof of Theorem 1.1}

4.1. Step 1: pointwise estimates. The discussion in section 3 made it clear that the natural objects for applying Birkhoff's ergodic theorem are functions involving a finite number (two in this case) of the $d_{n}$ 's with $n \rightarrow+\infty$. In this first step, we shall reduce the function $\psi_{r}$ to an expression of this form modulo terms that are small in some appropriate sense in the asymptotic regime that we consider - ie. as $t^{*} \rightarrow+\infty$.

Throughout this subsection, we set $r \in\left(0, \frac{1}{2}\right)$ and $\theta \in\left(0, \frac{\pi}{4}\right)$ such that $\alpha=\tan \theta \notin \mathbf{Q}$; let then $v=(\cos \theta, \sin \theta)$ and $R=\frac{r}{\cos \theta}$. We also consider $n \geq 1$ and $k \in\left\{1, \ldots, a_{n}\right\}$ (where $\alpha=\left[a_{1}, a_{2} \ldots\right]$ ) such that $R \in I_{n, k}$ - this defines $n$ and $k$ in a unique way, since the $I_{n, k}$ 's form a partition of $(0,1)$.

Lemma 4.1. Under these conditions on $\alpha, n k$ and $R$

- the integer $k$ is given by the formula

$$
k=\inf \left\{l \in \mathbf{N}^{*} \mid d_{n-1}-l d_{n} \leq R\right\}=\left[\frac{d_{n-1}-R}{d_{n}}\right]+1
$$


- the denominator $q_{n}$ of the $n$-th convergent of $\alpha$ satisfies the estimate

$$
\frac{1}{R+(k+1) d_{n}}<q_{n}<\frac{1}{R+(k-1) d_{n}}
$$

- finally, one has

$$
q_{n} d_{n}<\frac{1}{k}, \quad 0<1-q_{n} d_{n-1}<\frac{2}{k+1} .
$$

Proof. Whenever $d_{n} \leq R<d_{n-1}$ - ie. whenever $R \in I_{n}$ - the condition $R \in I_{n, k}$ amounts to defining $k$ by the formula

$$
k=\inf \left\{l \in \mathbf{N}^{*} \mid d_{n-1}-l d_{n} \leq R\right\}=\left[\frac{d_{n-1}-R}{d_{n}}\right]+1 .
$$

Next we estimate $q_{n}$. Since $R \in I_{n, k}$, one has in particular the inequalities

$$
d_{n-1}-k d_{n} \leq R<d_{n-1}-d_{n}(k-1)
$$

which imply

$$
\frac{1}{R+d_{n}(k-1)}>\frac{1}{d_{n-1}}>q_{n}
$$

by the second inequality in (7.9). This is exactly the upper bound in (4.2). By (7.7),

$$
\begin{aligned}
1-R q_{n}-d_{n}(k-1) q_{n} & =d_{n} q_{n-1}+d_{n-1} q_{n}-R q_{n}-d_{n}(k-1) q_{n} \\
& <q_{n}\left(d_{n}+d_{n-1}-R-(k-1) d_{n}\right) \\
& <q_{n}\left(d_{n}+d_{n-1}-d_{n-1}+k d_{n}-(k-1) d_{n}\right) \\
& =2 d_{n} q_{n}
\end{aligned}
$$

which gives the lower bound in (4.2).

The upper bound in (4.2) and the fact that $R \geq d_{n}$ ( since $R \in I_{n, k}$ ) imply the first inequality in (4.3). As for the second inequality there, observe that $q_{n} d_{n-1} \leq 1$ by (17.9) which establishes the lower bound, while the upper bound follows from (4.2) in the following manner:

$$
\begin{aligned}
\frac{1}{d_{n-1}}-q_{n} & \leq \frac{R+d_{n}(k+1)-d_{n-1}}{d_{n-1}\left(R+d_{n}(k+1)\right)} \\
& <\frac{2 d_{n}}{d_{n-1}\left(R+d_{n}(k+1)\right)}<\frac{2}{d_{n-1}(k+1)}
\end{aligned}
$$

(where the penultimate inequality above follows from (4.4)). 
Lemma 4.2. Let $v, \alpha, n, k$ and $R$ be chosen as above, and let $t^{*}>2$. Define

$$
\chi_{r}\left(\frac{t^{*}}{r}, v\right)=\left(1-\frac{R}{d_{n-1}}-t^{*} \frac{d_{n}}{R}\right)_{+} ;
$$

then for $\frac{t^{*}}{R} \geq q_{n}$ we have

$$
\left|\psi_{r}\left(\frac{t^{*}}{r}, v\right)-\chi_{r}\left(\frac{t^{*}}{r}, v\right)\right| \leq \frac{4}{k} \mathbf{1}_{k \geq t^{*}-2} .
$$

Proof. Let $t^{*}>2$. In view of the second inequality in (7.9)

$$
R<d_{n-1}<\frac{1}{q_{n}}, \quad \text { so that } q_{n}<\frac{1}{R}<\frac{t^{*}}{R} .
$$

Next we compare the part of the graph of $t \mapsto \psi_{r}(t, v)$ that corresponds to $t \cos \theta \geq q_{n}$ with the straight line $\Gamma$ as defined on figure 5. They only differ when $q_{n-1}+k q_{n}<t \cos \theta<q_{n-1}+(k+1) q_{n}$, and since $\psi_{r}$ is a non increasing function, they differ by at most

$$
\psi_{r}\left(\frac{q_{n-1}+k q_{n}}{\cos \theta}, v\right)=\left(\left(d_{n-1}-(k-1) d_{n}\right)-R\right) q_{n} .
$$

By (4.4),

$$
0<\left[d_{n-1}-(k-1) d_{n}\right]-R<d_{n}
$$

Hence

$$
\begin{aligned}
0 & \leq \psi_{r}\left(\frac{t^{*}}{r}, v\right)-\left(1-R q_{n}-\left(\frac{t^{*}}{R}-q_{n}\right) d_{n}\right)_{+} \\
& \leq \psi_{r}\left(\frac{q_{n-1}+k q_{n}}{\cos \theta}, v\right) \mathbf{1}_{q_{n-1}+k q_{n}<\frac{t^{*}}{R}<q_{n-1}+(k+1) q_{n}} \\
& \leq q_{n} d_{n} \mathbf{1}_{t^{*}<(k+2) q_{n} R} \leq \frac{1}{k} \mathbf{1}_{k \geq t^{*}-2},
\end{aligned}
$$

where the last inequality follows from (4.3) and (4.7).

Next we estimate the difference

$$
\left(1-R q_{n}-\left(\frac{t^{*}}{R}-q_{n}\right) d_{n}\right)_{+}-\left(1-\frac{R}{d_{n-1}}-t^{*} \frac{d_{n}}{R}\right)_{+}
$$

Because of the second inequality in (7.9), this difference is nonnegative. Because the map $x \mapsto x_{+}$is a contraction, this difference is less than

$$
\frac{R}{d_{n-1}}-R q_{n}+q_{n} d_{n}
$$


on the other hand both terms in the difference above vanish whenever $\frac{t^{*}}{R}>q_{n-1}+(k+1) q_{n}$. Hence

$$
\begin{array}{r}
\left|\left(1-R q_{n}-\left(\frac{t^{*}}{R}-q_{n}\right) d_{n}\right)_{+}-\left(1-\frac{R}{d_{n-1}}-t^{*} \frac{d_{n}}{R}\right)_{+}\right| \\
\leq\left(\frac{R}{d_{n-1}}-R q_{n}+q_{n} d_{n}\right) \mathbf{1}_{\frac{t^{*}}{R} \leq q_{n-1}+(k+1) q_{n}} \\
\leq\left(\frac{2}{k+1}+\frac{1}{k}\right) \mathbf{1}_{\frac{t^{*}}{R} \leq q_{n-1}+(k+1) q_{n}} \leq \frac{3}{k} \mathbf{1}_{k \geq t^{*}-2}
\end{array}
$$

where the penultimate inequality rests on (4.3) and the fact that $R<$ $d_{n-1}$.

4.2. Step 2: applying the ergodic theorem. In this subsection again, we set $r \in\left(0, \frac{1}{2}\right)$ and pick $\theta \in\left(0, \frac{\pi}{4}\right)$ such that $\alpha=\tan \theta \notin$ Q; again we set $v=(\cos \theta, \sin \theta)$ and $R=\frac{r}{\cos \theta}$. As in the previous subsection, we define $n \geq 1$ by the condition $R \in I_{n}$. In other words, we set $n=N(\alpha, R)$. With these assumptions, consider the expression $\chi_{r}\left(\frac{t^{*}}{R}, v\right)$ given by (4.5), ie.

$$
\begin{aligned}
\chi_{r}\left(\frac{t^{*}}{R}, v\right) & =\left(1-\frac{R}{d_{N(\alpha, R)-1}}-t^{*} \frac{d_{N(\alpha, R)}}{R}\right)_{+} \\
& =\left(1-e^{\Delta_{1}\left(\alpha, e^{-x}\right)}-t^{*} e^{-\Delta_{0}\left(\alpha, e^{-x}\right)}\right)_{+}
\end{aligned}
$$

with $x=-\ln R$ while $\Delta_{0}\left(\alpha, e^{-x}\right)$ and $\Delta_{1}\left(\alpha, e^{-x}\right)$ are defined as in (3.7).

Proposition 4.1. Let $R^{*} \in(0,1)$. Let $t^{*}>1$; then, for ae. $\theta \in\left(0, \frac{\pi}{4}\right)$ such that $\alpha=\tan \theta \notin \mathbf{Q}$

$$
\begin{aligned}
\frac{1}{|\ln \epsilon|} \int_{\epsilon}^{R^{*}} & \chi_{R \cos \theta}\left(\frac{t^{*}}{R}, v\right) \frac{d R}{R} \rightarrow \\
& \frac{12}{\pi^{2}} \int_{0}^{1}\left(\ln \frac{1+\sqrt{1-z}}{1-\sqrt{1-z}}-\sqrt{1-z}\right) \frac{d z}{4 t^{*}+z} \\
& +\frac{6}{\pi^{2}} \int_{0}^{1}\left(\frac{z}{1+\sqrt{1-z}}-\frac{z}{1-\sqrt{1-z}}\right) \frac{d z}{4 t^{*}+z}
\end{aligned}
$$

as $\epsilon \rightarrow 0^{+}$, where $v=(\cos \theta, \sin \theta)$. 
Proof. The proof is based upon applying Proposition 3.1 to the function $f$ defined by $f\left(z_{1}, z_{2}\right)=\left(1-e^{z_{2}}-t^{*} e^{-z_{1}}\right)_{+}$, since, for each $R^{*} \in(0,1)$

$$
\begin{aligned}
\frac{1}{|\ln \epsilon|} \int_{\epsilon}^{R^{*}} \chi_{R \cos \theta} & \left(\frac{t^{*}}{R}, v\right) \frac{d R}{R} \\
& =\frac{1}{|\ln \epsilon|} \int_{x^{*}}^{|\ln \epsilon|} f\left(\Delta_{0}\left(\alpha, e^{-x}\right), \Delta_{1}\left(\alpha, e^{-x}\right)\right) d x
\end{aligned}
$$

Starting from $f$, an elementary computation leads to $F$ defined for each $\xi \in(0,1)$ as in Proposition 3.1 by

$$
\begin{aligned}
F(\xi)=\int_{0}^{|\ln \xi|} f(|\ln \xi|-y,-y) d y & =\int_{0}^{|\ln \xi|}\left(1-e^{-y}-t^{*} \xi e^{y}\right)_{+} d y \\
& =\int_{1}^{1 / \xi}\left(\zeta-1-t^{*} \xi \zeta^{2}\right)_{+} \frac{d \zeta}{\zeta^{2}}
\end{aligned}
$$

Assuming that $t^{*}>1$ and $\xi \in(0,1)$, elementary computations show that

$$
\begin{aligned}
& \zeta-1-t^{*} \xi \zeta^{2}<0 \text { for all } \zeta \in \mathbf{R} \text { if } 4 t^{*} \xi>1, \text { otherwise } \\
& \zeta-1-t^{*} \xi \zeta^{2} \geq 0 \text { iff } \frac{1-\sqrt{1-4 t^{*} \xi}}{2 t^{*} \xi} \leq \zeta \leq \frac{1+\sqrt{1-4 t^{*} \xi}}{2 t^{*} \xi}
\end{aligned}
$$

and hence

$$
\begin{aligned}
F(\xi) & =\int_{1}^{1 / \xi}\left(\zeta-1-t^{*} \xi \zeta^{2}\right)+\frac{d \zeta}{\zeta^{2}} \\
& =\int_{\frac{1-\sqrt{1-4 t^{*} \xi}}{2 t^{*} \xi}}^{\frac{1+\sqrt{1-4 t^{*} \xi}}{2 t^{*} \xi}}\left(\frac{1}{\zeta}-\frac{1}{\zeta^{2}}-t^{*} \xi\right) d \zeta \\
& =\ln \frac{1+\sqrt{1-4 t^{*} \xi}}{1-\sqrt{1-4 t^{*} \xi}}-\sqrt{1-4 t^{*} \xi} \\
& +\frac{2 t^{*} \xi}{1+\sqrt{1-4 t^{*} \xi}}-\frac{2 t^{*} \xi}{1-\sqrt{1-4 t^{*} \xi}} .
\end{aligned}
$$


Therefore

$$
\begin{aligned}
& \frac{12}{\pi^{2}} \int_{0}^{1} \frac{F(\xi) d \xi}{1+\xi} \\
& =\frac{12}{\pi^{2}} \int_{0}^{1 / 4 t^{*}}\left(\ln \frac{1+\sqrt{1-4 t^{*} \xi}}{1-\sqrt{1-4 t^{*} \xi}}-\sqrt{1-4 t^{*} \xi}\right) \frac{d \xi}{1+\xi} \\
& +\frac{12}{\pi^{2}} \int_{0}^{1 / 4 t^{*}}\left(\frac{2 t^{*} \xi}{1+\sqrt{1-4 t^{*} \xi}}-\frac{2 t^{*} \xi}{1-\sqrt{1-4 t^{*} \xi}}\right) \frac{d \xi}{1+\xi} \\
& =\frac{12}{\pi^{2}} \int_{0}^{1}\left(\ln \frac{1+\sqrt{1-z}}{1-\sqrt{1-z}}-\sqrt{1-z}\right) \frac{d z}{4 t^{*}+z} \\
& +\frac{6}{\pi^{2}} \int_{0}^{1}\left(\frac{z}{1+\sqrt{1-z}}-\frac{z}{1-\sqrt{1-z}}\right) \frac{d z}{4 t^{*}+z}
\end{aligned}
$$

4.3. Step 3: $L^{1}$ estimate of the remainder. The last ingredient in the proof of Theorem 1.1 consists in estimating the right hand side of (4.6) in average (integrating over the angle $\theta$ ).

Let $R \in(0,1)$ and $\alpha \in(0,1) \backslash \mathbf{Q}$; we define $n$ and $k$ by the condition $R \in I_{n, k}$ - below, this value of $k$ is denoted by $k(\alpha, R)$. Equivalently,

$$
n=N(\alpha, R)+1, \quad k(\alpha, R)=\left[\frac{d_{N(\alpha, R)}(\alpha)-R}{d_{N(\alpha, R)+1}(\alpha)}\right]+1 .
$$

Lemma 4.3. Let $m \equiv m(\theta) \in L^{\infty}\left(\left(0, \frac{\pi}{4}\right)\right)$. Then, for each $R \in(0,1)$ and each $\lambda>1$, one has

$$
\int_{0}^{\pi / 4} \mathbf{1}_{k(\tan \theta, R)>\lambda} m(\theta) d \theta \leq \frac{2\|m\|_{L^{\infty}}}{\lambda-1} .
$$

Proof. The definition of $k(\alpha, R)$ implies in particular that

$$
k(\alpha, R) \leq \frac{d_{N(\alpha, R)}(\alpha)}{d_{N(\alpha, R)+1}(\alpha)}+1=\frac{1}{T^{N(\alpha, R)} \alpha}+1 .
$$

Hence, for each $\lambda>1$, one has

$$
\begin{aligned}
d g-\operatorname{meas} & (\{\alpha \in(0,1) \mid k(\alpha, R) \geq \lambda\}) \\
& \leq d g-\operatorname{meas}\left(\left\{\alpha \in(0,1) \mid 0<T^{N(\alpha, R)} \alpha \leq \frac{1}{\lambda-1}\right\}\right) \\
& \leq d g-\text { meas }\left(\left(0, \frac{1}{\lambda-1}\right]\right)=\frac{1}{\ln 2} \ln \left(\frac{\lambda}{\lambda-1}\right) \rightarrow 0
\end{aligned}
$$

as $\lambda \rightarrow+\infty$. Changing variables from $\alpha$ to $\theta=\arctan \alpha$ and using the classical inequality $\ln (1+z) \leq z$ leads to (4.9). 
4.4. Step 4: end of the proof. We conclude the proof of Theorem 1.1 by bringing together the various ingredients described above. Let $m \in L^{\infty}\left(\left[0, \frac{\pi}{4}\right]\right)$ such that $m \geq 0$ and $\int_{0}^{\pi / 4} m(\theta) d \theta=1$.

By Lemma 4.2 - and especially the inequality (4.6) there - and Lemma 4.3, one has

$$
\begin{aligned}
\mid \frac{1}{\ln \epsilon} \int_{\epsilon}^{\epsilon^{*}} \int_{0}^{\pi / 4} & \psi_{r}\left(\frac{t^{*}}{r},(\cos \theta, \sin \theta)\right) m(\theta) d \theta \frac{d r}{r} \\
& -\frac{1}{\ln \epsilon} \int_{\epsilon}^{\epsilon^{*}} \int_{0}^{\pi / 4} \chi_{r}\left(\frac{t^{*}}{r},(\cos \theta, \sin \theta)\right) m(\theta) d \theta \frac{d r}{r} \mid \\
& \leq \frac{4}{t^{*}-2} \frac{1}{|\ln \epsilon|} \int_{\epsilon}^{\epsilon^{*}} \int_{0}^{\pi / 4} \mathbf{1}_{k(\tan \theta, r / \cos \theta)>t^{*}-2} m(\theta) d \theta \frac{d r}{r} \\
& \leq \frac{8\|m\|_{L^{\infty}}}{\left(t^{*}-3\right)^{2}}
\end{aligned}
$$

By Proposition 4.1 and dominated convergence,

$$
\frac{1}{|\ln \epsilon|} \int_{\epsilon}^{\epsilon^{*}} \int_{0}^{\pi / 4} \chi_{r}\left(\frac{t^{*}}{r},(\cos \theta, \sin \theta)\right) m(\theta) d \theta \frac{d r}{r} \rightarrow \Lambda\left(t^{*}\right)
$$

where

$$
\begin{aligned}
\Lambda\left(t^{*}\right) & =\frac{12}{\pi^{2}} \int_{0}^{1}\left(\ln \frac{1+\sqrt{1-z}}{1-\sqrt{1-z}}-\sqrt{1-z}\right) \frac{d z}{4 t^{*}+z} \\
& +\frac{6}{\pi^{2}} \int_{0}^{1}\left(\frac{z}{1+\sqrt{1-z}}-\frac{z}{1-\sqrt{1-z}}\right) \frac{d z}{4 t^{*}+z},
\end{aligned}
$$

as $\epsilon \rightarrow 0^{+}$. Hence

$$
\limsup _{\epsilon \rightarrow 0^{+}} \frac{1}{|\ln \epsilon|} \int_{\epsilon}^{\epsilon^{*}} \int_{0}^{\pi / 4} \psi_{r}\left(\frac{t^{*}}{r},(\cos \theta, \sin \theta)\right) m(\theta) d \theta \frac{d r}{r} \leq \Lambda\left(t^{*}\right)+\frac{8\|m\|_{L^{\infty}}}{\left(t^{*}-3\right)^{2}},
$$

while

$\liminf _{\epsilon \rightarrow 0^{+}} \frac{1}{|\ln \epsilon|} \int_{\epsilon}^{\epsilon^{*}} \int_{0}^{\pi / 4} \psi_{r}\left(\frac{t^{*}}{r},(\cos \theta, \sin \theta)\right) m(\theta) d \theta \frac{d r}{r} \geq \Lambda\left(t^{*}\right)-\frac{8\|m\|_{L^{\infty}}}{\left(t^{*}-3\right)^{2}}$.

As $t^{*} \rightarrow+\infty$, one has

$$
\begin{aligned}
\Lambda\left(t^{*}\right) & \sim \frac{3}{\pi^{2} t^{*}} \int_{0}^{1}\left(\ln \frac{1+\sqrt{1-z}}{1-\sqrt{1-z}}-\sqrt{1-z}\right) d z \\
& +\frac{3}{2 \pi^{2} t^{*}} \int_{0}^{1}\left(\frac{z}{1+\sqrt{1-z}}-\frac{z}{1-\sqrt{1-z}}\right) d z=\frac{2}{\pi^{2} t^{*}} .
\end{aligned}
$$


Since the function $t \mapsto \psi_{r}(t, v)$ is nonincreasing for all $v \in S^{1}$ and $r \in\left(0, \frac{1}{2}\right)$, one has, by using (2.16),

$$
\begin{aligned}
& \limsup _{\epsilon \rightarrow 0^{+}} \frac{1}{|\ln \epsilon|} \int_{\epsilon}^{\epsilon^{*}} \int_{0}^{\pi / 4} \phi_{r}\left(\frac{t^{*}}{r},(\cos \theta, \sin \theta)\right) m(\theta) d \theta \frac{d r}{r} \\
= & \limsup _{\epsilon \rightarrow 0^{+}} \frac{1}{|\ln \epsilon|} \int_{\epsilon}^{2 s} \int_{0}^{\pi / 4} \phi_{r}\left(\frac{t^{*}}{r},(\cos \theta, \sin \theta)\right) m(\theta) d \theta \frac{d r}{r} \\
& \leq \limsup _{\epsilon \rightarrow 0^{+}} \frac{1}{|\ln \epsilon|} \int_{\epsilon}^{2 s} \int_{0}^{\pi / 4} \psi_{r}\left(\frac{t^{*}-\frac{r}{2}}{r},(\cos \theta, \sin \theta)\right) m(\theta) d \theta \frac{d r}{r} \\
\leq & \limsup _{\epsilon \rightarrow 0^{+}} \frac{1}{|\ln \epsilon|} \int_{\epsilon}^{2 s} \int_{0}^{\pi / 4} \psi_{r}\left(\frac{t^{*}-s}{r},(\cos \theta, \sin \theta)\right) m(\theta) d \theta \frac{d r}{r} \\
\leq & \Lambda\left(t^{*}-s\right)+\frac{8\|m\|_{L^{\infty}}}{\left(t^{*}-s-3\right)^{2}},
\end{aligned}
$$

for each $s \in\left(0, \frac{1}{2}\right)$. Letting $s \rightarrow 0^{+}$in the last inequality, one arrives at

$$
\begin{array}{r}
\limsup _{\epsilon \rightarrow 0^{+}} \frac{1}{|\ln \epsilon|} \int_{\epsilon}^{\epsilon^{*}} \int_{0}^{\pi / 4} \phi_{r}\left(\frac{t^{*}}{r},(\cos \theta, \sin \theta)\right) m(\theta) d \theta \frac{d r}{r} \\
\leq \Lambda\left(t^{*}\right)+\frac{8\|m\|_{L^{\infty}}}{\left(t^{*}-3\right)^{2}} .
\end{array}
$$

A similar inequality holds for the lim inf. By symmetry, the averaging in $\theta$ in (4.10) can be done equivalently on $\left(0, \frac{\pi}{4}\right)$ or in $(0,2 \pi)$, which eventually proves (1.9).

\section{Applichtions to kinetic theory}

It has been proved in Theorem 2.1 of [12] (see also [6]) that the linear Boltzmann equation (ie. equation (10) of [13]) does not govern the Boltzmann-Grad limit of the periodic Lorentz gas — unlike the case of a Lorentz gas with a random (Poisson) distribution of scatterers, where the linear Boltzmann equation was rigorously derived in [1] and [5]. While Theorem 2.1 of [12] is merely a negative result, we show below how to infer from Proposition 1.1 positive information on the asymptotic behavior of the periodic Lorentz gas in the BoltzmannGrad limit.

Define $\Omega_{\epsilon}=\left\{\epsilon z \mid z \in Z_{\epsilon}\right\}$, and consider the transport equation

$$
\begin{aligned}
\partial_{t} f_{\epsilon}+v \cdot \nabla_{x} f_{\epsilon} & =0, \quad x \in \Omega_{\epsilon},|v|=1 \\
f_{\epsilon}(t, x, v) & =0, \quad x \in \partial \Omega_{\epsilon}, v \cdot n_{x}>0, \\
f_{\epsilon}(0, x, v) & =f^{i n}(x, v) \quad x \in \Omega_{\epsilon},|v|=1 .
\end{aligned}
$$


Here, the unknown is $f_{\epsilon} \equiv f_{\epsilon}(t, x, v)$ while $n_{x}$ is the inward unit normal at point $x \in \partial \Omega_{\epsilon}$ and $f^{i n}$ is a given, nonnegative function of $C_{c}\left(\mathbf{R}^{2} \times \mathbf{S}^{1}\right)$. Physically, this is a variant of the periodic Lorentz gas where scatterers are replaced by holes (or traps) where impinging particles fall and thus are removed from the domain $\Omega_{\epsilon}$. Obviously, for each $t \geq 0$

$$
\left\|f_{\epsilon}\right\|_{L_{t, x, v}^{\infty}}=\left\|f^{i n}\right\|_{L_{x, v}^{\infty}} .
$$

Reasoning as in [13] suggests that $f_{\epsilon} \rightarrow f$ in $L_{t, x, v}^{\infty}$ weak-* where $f$ solves the uniformly damped transport equation

$$
\partial_{t} f+v \cdot \nabla_{x} f+f=0 \text { on } \mathbf{R}_{+}^{*} \times \mathbf{R}^{2} \times \mathbf{S}^{1}, \quad f_{\mid t=0}=f^{i n},
$$

but this is ruled out by Theorem 2.1 of [12. Instead, Proposition 1.1] suggests that the resulting damping rate should vanish in the limit as $t \rightarrow+\infty$. This statement is made precise in the following theorem.

Theorem 5.1. Let $f^{\text {in }} \geq 0$ belong to $C_{c}\left(\mathbf{R}^{2} \times \mathbf{S}^{1}\right)$ and let $f_{\epsilon}$ be, for each $\epsilon \in\left(0, \frac{1}{4}\right)$, the solution of (5.1). Then, for each nonnegative test function $\chi \in C_{c}^{1}\left(\mathbf{R}^{2} \times \mathbf{S}^{1}\right)$, one has

$$
\begin{array}{r}
\limsup _{\epsilon \rightarrow 0} \iint\left(\frac{1}{|\ln \epsilon|} \int_{\epsilon}^{1 / 4} f_{r}(t, x, v) \frac{d r}{r}\right) \chi(x, v) d x d v \\
=\iint f(t, x, v) \chi(x, v) d x d v+O\left(\frac{1}{t^{2}}\right) \\
\liminf _{\epsilon \rightarrow 0} \iint\left(\frac{1}{|\ln \epsilon|} \int_{\epsilon}^{1 / 4} f_{r}(t, x, v) \frac{d r}{r}\right) \chi(x, v) d x d v \\
=\iint f(t, x, v) \chi(x, v) d x d v+O\left(\frac{1}{t^{2}}\right)
\end{array}
$$

as $t \rightarrow+\infty$, where

$$
f(t, x, v)=\frac{2 f^{i n}(x-t v, v)}{\pi^{2} t} .
$$

In particular, $f$ satisfies

$$
\partial_{t} f+v \cdot \nabla_{x} f+\frac{1}{t} f=0, \quad(t, x, v) \in(0,+\infty) \times \mathbf{R}^{2} \times \mathbf{S}^{1}
$$

in the sense of distributions.

Proof. First the solution of (5.1) is given by the formula

$$
f_{\epsilon}(t, x, v)=f^{i n}(x-t v, v) \mathbf{1}_{\tau_{\epsilon}(x / \epsilon,-v) \geq t / \epsilon} .
$$

In this formula, the exit time $\tau_{\epsilon}$ is considered as a function defined on $Z_{\epsilon} \times \mathbf{S}^{1}$ with $\mathbf{Z}^{2}$-periodicity and extended by 0 in $Z_{\epsilon}^{c} \times \mathbf{S}^{1}$. 
Let $h \equiv h(t, x, v) \geq 0$ belong to $C^{\infty}\left(\mathbf{R}_{+} \times \mathbf{R}^{2} \times \mathbf{S}^{1}\right)$, with support in $\mathbf{R}_{+} \times[-L, L]^{2} \times \mathbf{S}^{1}$

$$
\begin{array}{r}
\left|\iint h(t, x, v) \mathbf{1}_{\tau_{r}\left(\frac{x}{r},-v\right) \geq \frac{t}{r}} d x d v-\int_{\mathbf{S}^{1}} \phi_{r}\left(\frac{t}{r}, v\right) \int h(t, x, v) d x d v\right| \\
\leq\left|\iint h(t, x, v) \mathbf{1}_{\tau_{\epsilon}\left(\frac{x}{r},-v\right) \geq \frac{t}{r}} d x d v-r^{2} \sum_{l \in \mathbf{Z}^{2}} \int_{\mathbf{S}^{1}} h(t, r l, v) \int_{\mathbf{T}^{2}} \mathbf{1}_{\tau_{r}(y,-v) \geq \frac{t}{r}} d y d v\right| \\
\quad+\int_{\mathbf{S}^{1}} \phi_{r}\left(\frac{t}{r}, v\right)\left|r^{2} \sum_{l \in \mathbf{Z}^{2}} h(t, r l, v)-\int h(t, x, v) d x\right| d v \\
=r^{2} \sum_{l \in \mathbf{Z}^{2}} \iint_{\mathbf{T}^{2} \times \mathbf{S}^{1}}|h(t, r l+r y, v)-h(t, r l, v)| \mathbf{1}_{\tau_{r}(y,-v) \geq \frac{t}{r}} d y d v \\
+r^{2} \sum_{l \in \mathbf{Z}^{2}} \iint_{\mathbf{T}^{2} \times \mathbf{S}^{1}}|h(t, r l+r y, v)-h(t, r l, v)| \phi_{r}\left(\frac{t}{r}, v\right) d y d v \\
\leq 2 r\left\|\nabla_{x} h\right\|_{L^{\infty}}\left|S^{1}\right| \cdot r^{2} \sum_{l \in \mathbf{Z}^{2}} \mathbf{1}_{[-L, L]^{2}}(r l) \\
\leq 2 r\left\|\nabla_{x} h\right\|_{L^{\infty}}\left|S^{1}\right|\left(L^{2}+o(1)\right) .
\end{array}
$$

By Theorem 1.1

$$
\begin{aligned}
\mid \limsup _{\epsilon \rightarrow 0^{+}} \frac{1}{|\ln \epsilon|} \int_{\epsilon}^{\epsilon^{*}} \int_{\mathbf{S}^{1}} \phi_{r}\left(\frac{t}{r}, v\right) & \int h(t, x, v) d x d v \frac{d r}{r} \\
& -\frac{2}{\pi^{2} t} \iint h(t, x, v) d x d v \mid \\
\leq & \frac{8\|h\|_{L_{t, v}^{\infty}\left(L_{x}^{1}\right)}}{t^{*}-3}
\end{aligned}
$$

with a similar estimate for the liminf.

Putting together (5.7), (5.8) with the formula (5.6) establishes (5.4).

Theorem 5.1 can also be viewed as a result in homogenization. This remark leads to a comparison with the analogous situation - homogenization of a diffusion process with Dirichlet boundary conditions in $Z_{r}$ - studied in [10] (in truth, the result obtained in [10] is much more complete and satisfying than Theorem [5.1). Not surprisingly, the mathematical tools used in [10] are of a very different nature than the ones in the present work. This however is by no means surprising and simply reflects the very different nature of the trajectories of a diffusion process and of those of a free transport equation. It is very likely that 
the nature of the result in Theorem 5.1 — and its proof — would be deeply affected by adding some collision (ie. jump in velocity) process to the free transport between successive impingements on the obstacles.

\section{Final REMARKS AND PERSPECTIVES}

A first question left open in the present work is the existence of the limit (1.7). As the reader will have probably noticed in the proof of Theorem 1.1. the diameter $r$ of the obstacle is treated as a time variable under renormalization - ie. transformation of the problem by the Gauss map. It may be that new insight concerning (1.7) can be gained by using more specific properties of $T$ than ergodicity as in the present paper.

A second question is the existence of the limit in the sense of Cesaro, as commented upon in Remark 1.1. As can be seen from the proof of Theorem 1.1, proving this essentially amounts to being able to apply an ergodic theorem as in section 3 to functions of the form

$$
f\left(\epsilon q_{N(\alpha, \epsilon)}, \frac{d_{N}(\alpha, \epsilon)}{\epsilon}, \frac{d_{N(\alpha, \epsilon)-1}}{\epsilon}\right) .
$$

(In the present paper, (4.3) essentially allows one to replace $q_{N(\alpha, \epsilon)}$ by $\left.1 / d_{N(\alpha, \epsilon)-1}\right)$. This extension of the ergodic theorem in the present paper is postponed to a subsequent paper [8] and will be applied to the problem of the Lyapunov exponent for the Lorentz gas (see 9] for a presentation of this subject).

Finally we would like comment on some very interesting, related work in [3] and 4].

The billiard problem considered in [3] is similar to studying the distribution of free path lengths in $Z_{r}$ with the obstacle at the origin removed (ie. in $\left.Z_{r} \cup \overline{B(0, r / 2)}\right)$ for particles starting from the origin only. This is a quite different problem and thus the limit as $r \rightarrow 0^{+}$of this distribution has nothing to do with the simple result in Theorem 1.1. A related issue is studied in [4]: for $R>0$, consider the directions of lattice points (ie. point of $\mathbf{Z}^{2}$ ) in the ball $B(0, R)$ of $\mathbf{R}^{2}$. These directions make a set of $N_{R}$ angles

$$
0 \leq \theta_{0}<\theta_{1}, \ldots<\theta_{N_{R}}=2 \pi ;
$$

the paper 4] computes the distribution of scaled differences of the form $N_{R}\left(\theta_{k+1}-\theta_{k}\right)$ as $R \rightarrow+\infty$. Remarkably, these differences are far from being exponentially distributed - unlike in the case of angles picked at random in $[0,2 \pi)$. Consistently with the results in [6] and in [12, this observation stresses again the difference between the case 
of a random distribution of scatterers as studied in 11] and that of a periodic distribution scatterers.

\section{ApPEndix:}

\section{BACKGROUND ON CONTINUED FRACTIONS}

We recall below some basic facts and notations about continued fractions. Given $\alpha \in(0,1)$, the Gauss map is defined by

$$
T \alpha=\frac{1}{\alpha}-\left[\frac{1}{\alpha}\right]
$$

it is known to be a uniquely ergodic transformation of $(0,1)$ with invariant measure

$$
d g(\alpha)=\frac{1}{\ln 2} \frac{d \alpha}{1+\alpha} .
$$

The continued fraction expansion of $\alpha$ is

$$
\alpha=\left[a_{1}, a_{2}, a_{3}, \ldots\right]=\frac{1}{a_{1}+\frac{1}{a_{2}+\frac{1}{a_{3}+\ldots}}} \text { with } a_{k}=\left[\frac{1}{T^{k-1} \alpha}\right], k \geq 1 ;
$$

and the action of $T$ is seen to correspond to the shift

$$
\alpha=\left[a_{1}, a_{2}, a_{3}, \ldots\right] \mapsto T \alpha=\left[a_{2}, a_{3}, a_{4}, \ldots\right] .
$$

The convergents of $\alpha$ are defined by the recursion formulas

$$
\begin{aligned}
& q_{n+1}=a_{n} q_{n}+q_{n-1}, \quad q_{0}=0, q_{1}=1, \\
& p_{n+1}=a_{n} p_{n}+p_{n-1}, \quad p_{0}=1, p_{1}=0,
\end{aligned}
$$

and the corresponding error $d_{n}=\left|q_{n} \alpha-p_{n}\right|$ satisfies

$$
d_{n+1}=-a_{n} d_{n}+d_{n-1}, \quad d_{0}=1, d_{1}=\alpha .
$$

The first relation in (7.5) and (7.6) imply that

$$
q_{n} d_{n+1}+q_{n+1} d_{n}=1, \quad n \in \mathbf{N}^{*} .
$$

For each $\alpha \in(0,1) \backslash \mathbf{Q}$, the convergents of $\alpha$ are the sequence of best rational approximants of $\alpha$. In other words

$$
\left|q_{n} \alpha-p_{n}\right|=\inf \left\{|q \alpha-p| \mid p, q \in \mathbf{Z}, 0 \leq q<q_{n}\right\} .
$$

This implies in particular that the approximation by continued fractions is alternate in the sense that the algebraic value of the error is

$$
q_{n} \alpha-p_{n}=(-1)^{n-1} d_{n}, \quad n \geq 0 .
$$


Also, for each $\alpha \in(0,1) \backslash \mathbf{Q}$, the sequence of errors satisfies the inequalities

$$
\frac{1}{q_{n}+q_{n+1}}<d_{n}<\frac{1}{q_{n+1}} .
$$

The notations $a_{n}(\alpha), p_{n}(\alpha), q_{n}(\alpha)$ and $d_{n}(\alpha)$ are used to emphasize the dependence of these quantities on $\alpha$ whenever necessary.

Our discussion uses "renormalization", ie. transforming $\alpha$ by the iterates of the Gauss map T. We have gathered some useful facts in the next lemma.

Lemma 7.1. Let $\alpha \in(0,1)$; then

- for each $n \in \mathbf{N}, q_{n}(T \alpha)=p_{n+1}(\alpha)$ and $d_{n+1}(\alpha)=\alpha d_{n}(T \alpha)$;

- for each $n \in \mathbf{N}$,

$$
d_{n}(\alpha)=\prod_{k=0}^{n-1} T^{k} \alpha
$$

Proof. First observe that

$$
a_{n}(T \alpha)=a_{n+1}(\alpha), \quad n \geq 1 .
$$

This relation and (7.5) implies that the sequences $q_{n}(T \alpha)$ and $p_{n+1}(\alpha)$ satisfy the same recursion formulae; thus in order to check the first formula, it suffices to check it for both $n=0$ and $n=1$ (in the latter case, one has $\left.q_{1}(T \alpha)=[1 / T \alpha]=a_{2}=p_{2}(\alpha)\right)$. The second formula is checked in the same way, and clearly implies the expression of $d_{n}(\alpha)$ in terms of the $T^{k} \alpha$ 's.

Acknowledgements. We thank Prof. H. S. Dumas who told us that using ref. [2] might simplify our original method for deriving formulas (2.10)-2.13). The research of E. C. has been partially supported by MIUR and by INDAM GNFM. Both authors acknowledge the support of the European Research Training Network HyKE (Contract no. HPRNCT-2002-00282).

\section{REFERENCES}

[1] V. I. Arnold Mathematical methods of classical mechanics, 2nd edition Springer Verlag, New York, 1989.

[2] S. Blank, N. Krikorian Thom's problem on irrational flows, Internat. J. of Math. 4 (1993), 721-726.

[3] F. Boca, R. Gologan, A. Zaharescu The statistics of the trajectory in a certain billiard in a flat two-torus, preprint, 2001.

[4] F. Boca, C. Cobeli, A. Zaharescu Distribution of lattice points visible from the origin, Commun. Math. Phys. 213 (2000), 433-570. 
[5] C. Boldrighini, L. Bunimovich, Ya. G. Sinai On the Boltzmann equation for the Lorentz gas, J. Statist. Phys. 32 (1983), 477-501.

[6] J. Bourgain, F. Golse, B. Wennberg On the Distribution of Free Path Lengths for the Periodic Lorentz Gas, Commun. Math. Phys. 190 (1998), 491-508.

[7] L. Bunimovich Billiards and other hyperbolic systems in Dynamical systems, ergodic theory and applications, Ya. G. Sinai and al. ed.; pp. 192-233, Encyclopaedia Math. Sci. 100, 2nd ed., Springer-Verlag, Berlin, 2000.

[8] E. Caglioti, F. Golse work in preparation.

[9] N. Chernov Entropy values and entropy bounds, in Hard ball systems and the Lorentz gas, D. Szász ed., pp. 121-143, Encyclopaedia Math. Sci., 101, Springer-Verlag, Berlin, 2000.

[10] D. Cioranescu, F. Murat Un terme étrange venu d'ailleurs I 83 II, in Nonlinear partial differential equations and their applications: Collège de France Seminar, I in Vol. II (Paris, 1979/1980), pp. 98-138, 389-390, II in Vol. III (Paris, 1980/1981), pp. 154-178, 425-426, Res. Notes in Math., 60 \& 70, Pitman, Boston, Mass.-London, 1982.

[11] G. Gallavotti Rigorous theory of the Boltzmann equation in the Lorentz gas, Nota interna no. 358, Istituto di Fisica, Univ. di Roma (1972). Reprinted in "Statistical mechanics: a short treatise", Springer, Berlin-Heidelberg (1999), pp. $48-55$

[12] F. Golse, B. Wennberg On the distribution of free path lengths for the periodic Lorentz gas II, M2AN Modél. Math. et Anal. Numér. 34, no. 6 (2000), 11511163.

[13] H. Lorentz Le mouvement des électrons dans les métaux, Arch. Néerl. 10 (1905), p. 336; reprinted in Collected papers, vol. 3 pp. 180-214, Martinus Nijhoff, The Hague 1936.

(E. C.) Dipartimento di Matematica, Istituto Guido Castelnuovo, Università di Roma "La Sapienza", p.le Aldo Moro 2, I00185 Roma

E-mail address: caglioti@mat.uniroma1.it

(F. G.) Institut Universitaire de France, \& Département de Mathématiques et Applications, Ecole Normale Supérieure Paris, 45 rue D'Ulm, F75230 PARIS CEDEX 05

E-mail address: golse@dma.ens.fr 